INJE-TP-01-04, hep-th/0104159

\title{
Holographic principle in the BDL brane cosmology
}

\author{
N. J. Kim* ${ }^{*}$ H. W. Lee ${ }^{\dagger}$, and Y.S. Myung ${ }^{\ddagger}$ \\ Department of Physics, Graduate School, Inje University, Kimhae 621-749, Korea \\ Gungwon Kang§ \\ KEK Theory Group, High Energy Accelerator Research Organization, 1-1 Oho, Tsukuba, Ibaraki \\ 305-0801, Japan
}

\begin{abstract}
We study the holographic principle in the brane cosmology. Especially we describe how to accommodate the 5D anti de Sitter Schwarzschild $\left(\mathrm{AdSS}_{5}\right)$ black hole in the Binetruy-Deffayet-Langlois (BDL) approach of brane cosmology. It is easy to make a connection between a mass $M$ of the $\mathrm{AdSS}_{5}$ black hole and a conformal field theory (CFT)-radiation dominated universe on the brane in the moving domain wall approach. But this is not established in the BDL approach. In this case we use two parameters $C_{1}, C_{2}$ in the Friedmann equation. These arise from integration and are really related to the choice of initial bulk matter. If one chooses a bulk energy density $\rho_{B}$ to account for a mass $M$ of the $\mathrm{AdSS}_{5}$ black hole and the static fifth dimension, a CFTradiation term with $\rho_{C F T} \sim M / a^{4}$ comes out from the bulk matter without introducing a localized matter distribution on the brane. This means that the holographic principle can be established in the BDL brane cosmology.
\end{abstract}

\footnotetext{
*E-mail address: dtpnjk@ijnc.inje.ac.kr

${ }^{\dagger}$ E-mail address: hwlee@physics.inje.ac.kr

$\ddagger$ E-mail address: ysmyung@physics.inje.ac.kr

§gwkang@post.kek.jp
} 
Recently there has been much interest in the phenomenon of localization of gravity proposed by Randall and Sundrum (RS) [1,2]. RS assumed a single positive tension 3-brane and a negative bulk cosmological constant in the five dimensional (5D) spacetime [2]. They have obtained a four dimensional (4D) localized gravity by fine-tuning the tension of the brane to the cosmological constant. On the other hand, several authors have studied its cosmological implications. The brane cosmology contains some important deviation from the Friedmann-Robertson-Walker (FRW) cosmology. One approach is first to assume the $5 \mathrm{D}$ dynamic metric (that is, BDL-metric [3, [4,6]) which is manifestly $Z_{2}$-symmetrid円. Then one solves the Einstein equation with a localized stress-energy tensor to find the behavior of the scale factors. We call this the BDL approach.

The other approach starts with a static configuration which is two-sided $\mathrm{AdSS}_{5}$ spaces joined by the domain wall. In this case the embedding into the MDW2 is possible by choosing a normal vector $n_{M}$ and a tangent vector $u_{M}$ [8]. The domain wall separating two bulk spaces is taken to be located at $r=a(\tau)$, where $a(\tau)$ will be found by solving the Israel junction condition [9]. In this picture an observer on the MDW will interpret his motion through the static bulk background as cosmological expansion or contraction. It was shown that two approaches can describe the same spacetime evolution [10,11].

More recently, the AdS/CFT correspondence has been studied in the context of MDW approach. This is one way of realizing the holographic principle. This principle was investigated in the brane cosmology with a CFT within one-sided $\mathrm{AdSS}_{5}$ black hole spacetime 112,13. It shows that the brane carries with the bulk information of the $\mathrm{AdSS}_{5}$ black hole.

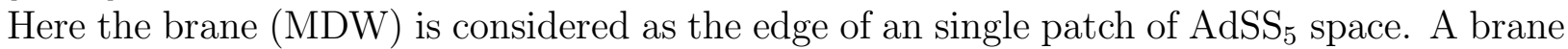
starts with (big bang) inside the small black hole $\left(\ell>r_{+}\right)$, crosses the horizon, and expands until it reaches maximum size. And then the brane contracts, it falls the black hole again and finally disappears (big crunch). An observer in $\mathrm{AdSS}_{5}$-space finds two interesting moments (see two points on the Penrose diagram in [10]) when the brane crosses the past (future) event horizon. Authors in ref. [14] showed that at these times the Friedmann equation controlling the cosmological expansion (contraction) coincides with the Cardy-Verlinde formula which is the entropy-energy relation for the $4 \mathrm{D}$ CFT on the brane. This is a CFT-Cosmology relation.

In this paper, we will show that the above holographic principle can be also realized within the BDL approach. It is easy to make connection between a mass $M$ of the $\operatorname{AdSS}_{5}$ black hole and a CFT-radiation dominated universe on the brane in the moving domain wall approach [12,13]. But as far as we know, this is not yet established in the BDL approach. In the BDL method we usually have two parameters $C_{1}, C_{2}$ in the Friedmann equation [4]. These result from integration and are really related to the choice of initial bulk matter $\left(\rho_{B}, P\right)$. Hence we will use this parameter to make a connection between the brane and the bulk. This is possible because the BDL metric can lead to the $\mathrm{AdSS}_{5}$ metric by a coordinate transformation $:\left\{\tau, x^{i}, y\right\} \rightarrow\{t, r, \chi, \theta, \phi\}$. We find a relation $C_{2}(0)=\kappa^{2} a_{0}^{4} \rho_{B} / 6$

\footnotetext{
${ }^{1}$ We call this two-sided brane world in comparison with one-sided brane world [7].

${ }^{2}$ Here we use the term "moving domain wall" loosely to refer to any 3-brane moving in 5 dimensions.
} 
which turn out to be constant on the brane in order to go well with other conditions, in addition to $C_{1}=0$. Considering both $C_{2}(0) \sim M$ and the static condition $\dot{b}=0$ for the fifth dimension, a CFT-radiation term with $\rho_{C F T} \sim M / a_{0}^{4}$ comes out from the bulk spacetime without introducing a localized matter distribution on the brane. Hence we call the BDL approach under certain conditions as the fixed domain wall approach, in compared with the MDW approach.

We start with the BDL metric for the brane cosmology [ [] ]

$$
d s^{2}=-n^{2}(\tau, y) d \tau^{2}+a^{2}(\tau, y) \gamma_{i j} d x^{i} d x^{j}+b^{2}(\tau, y) d y^{2}
$$

where $\gamma_{i j}$ is a maximally symmetric $3 \mathrm{D}$ space parametrized by $k \in\{-1,0,1\}$.

Then the 5D Einstein equation takes the form

$$
G_{A B} \equiv R_{A B}-\frac{1}{2} R g_{A B}=\kappa^{2} T_{A B}
$$

with $\kappa^{2}=8 \pi G_{5}^{N}$. The Einstein tensor is given by

$$
\begin{aligned}
& G_{00}=3\left\{\left(\frac{\dot{a}}{a}\right)^{2}+\frac{\dot{a} \dot{b}}{a b}-\frac{n^{2}}{b^{2}}\left(\frac{a^{\prime \prime}}{a}+\left(\frac{a^{\prime}}{a}\right)^{2}-\frac{a^{\prime} b^{\prime}}{a b}\right)+k \frac{n^{2}}{a^{2}}\right\}, \\
& G_{i j}=\frac{a^{2}}{b^{2}} \gamma_{i j}\left\{\left(\frac{a^{\prime}}{a}\right)^{2}+2 \frac{a^{\prime} n^{\prime}}{a n}-2 \frac{a^{\prime} b^{\prime}}{a b}-\frac{b^{\prime} n^{\prime}}{b n}+2 \frac{a^{\prime \prime}}{a}+\frac{n^{\prime \prime}}{n}\right\} \\
& +\frac{a^{2}}{n^{2}} \gamma_{i j}\left\{-\left(\frac{\dot{a}}{a}\right)^{2}+2 \frac{\dot{a} \dot{n}}{a n}-2 \frac{\dot{a} \dot{b}}{a b}+\frac{\dot{b} \dot{n}}{b n}-2 \frac{\ddot{a}}{a}+\frac{\ddot{b}}{b}\right\}-k \gamma_{i j}, \\
& G_{05}=3\left(\frac{\dot{a} n^{\prime}}{a n}+\frac{a^{\prime} \dot{b}}{a b}-\frac{\dot{a}^{\prime}}{a}\right), \\
& G_{55}=3\left\{\left(\frac{a^{\prime}}{a}\right)^{2}+\frac{a^{\prime} n^{\prime}}{a n}-\frac{b^{2}}{n^{2}}\left(\frac{\ddot{a}}{a}+\left(\frac{\dot{a}}{a}\right)^{2}-\frac{\dot{a} \dot{n}}{a n}\right)-k \frac{n^{2}}{a^{2}}\right\},
\end{aligned}
$$

where dot(prime) denote differentiations with respect to $\tau(y)$. And the matter source is given by the bulk and brane parts

$$
T_{B}^{A}=\left.\check{T}_{B}^{A}\right|_{\text {bulk }}+\left.\tilde{T}_{B}^{A}\right|_{\text {brane }}
$$

with the bulk stress-energy tensor distributed in whole space

$$
\left.\check{T}_{B}^{A}\right|_{\text {bulk }}=\left[-\left(\rho_{B}+\Lambda\right),-\Lambda+P,-\Lambda+P,-\Lambda+P,-\Lambda+P_{T}\right]
$$

and the localized matter on the brane at $y=0$

$$
\left.\tilde{T}_{B}^{A}\right|_{\text {brane }}=\frac{\delta(y)}{b}\left[-\left(\sigma+\rho_{b}\right),-\sigma+p_{b},-\sigma+p_{b},-\sigma+p_{b}, 0\right] .
$$

Here we split the bulk tensor into the cosmological constant $\Lambda$ and the bulk matter $\left(\rho_{B}, P, P_{T}\right)$ for convenience. The brane tension $\sigma$ is introduced for the fine-tuning. Also we assume that all of matters are independent of the fifth coordinate $y$. Further we include the different bulk pressure $P_{T}$ along the fifth direction for general discussion. The assumption of $\tilde{T}_{5}^{0}=0$ which means that there is no flow of matter along the fifth direction implies that $G_{05}=0$. Let us introduce $F$ to solve the Einstein equation as 


$$
F \equiv \frac{\left(a a^{\prime}\right)^{2}}{b^{2}}-\frac{(a \dot{a})^{2}}{n^{2}}-k a^{2}
$$

Then we note that two components of Einstein equation take the forms

$$
\begin{aligned}
& G_{00}=-\frac{3}{2} \frac{n^{2}}{a^{3} a^{\prime}} F^{\prime}=\kappa^{2} T_{00} \\
& G_{55}=\frac{3}{2} \frac{b^{2}}{a^{3} \dot{a}} \dot{F}=\kappa^{2} T_{55}
\end{aligned}
$$

which imply

$$
\begin{aligned}
& F^{\prime}=-\frac{2}{3} \frac{a^{3} a^{\prime}}{n^{2}} \kappa^{2} T_{00}=-\frac{2}{3} \kappa^{2} a^{3} a^{\prime}\left(\Lambda+\rho_{B}+\frac{1}{b}\left(\sigma+\rho_{b}\right) \delta(y)\right), \\
& \dot{F}=\frac{2}{3} \frac{a^{3} \dot{a}}{b^{2}} \kappa^{2} T_{55}=-\frac{2}{3} \kappa^{2} a^{3} \dot{a}\left(\Lambda-P_{T}\right) .
\end{aligned}
$$

In the bulk of which is everywhere except $y \neq 0$, Eqs.(10) and (11) lead to

$$
\begin{aligned}
& F^{\prime}=-\frac{2}{3} \kappa^{2} a^{3} a^{\prime}\left(\Lambda+\rho_{B}\right) \\
& \dot{F}=-\frac{2}{3} \kappa^{2} a^{3} \dot{a}\left(\Lambda-P_{T}\right)
\end{aligned}
$$

Integrating Eq.(12) over $y$ and Eq.(13) over $\tau$ give us two integration constants $C_{1}(\tau)$ and $C_{2}(y)^{3}$

$$
\begin{aligned}
& F=-\frac{1}{6} \kappa^{2} a^{4}\left(\Lambda+\rho_{B}\right)-C_{1}(\tau), \\
& F=-\frac{1}{6} \kappa^{2} a^{4}\left(\Lambda-P_{T}\right)-\frac{1}{4} \int a^{4} \dot{P}_{T} d \tau-C_{2}(y)
\end{aligned}
$$

which combine to find out the relation between $C_{1}(\tau)$ and $C_{2}(y)$

$$
C_{2}(y)=\frac{1}{6} \kappa^{2} a^{4}\left(\rho_{B}+P_{T}\right)-\frac{1}{4} \int a^{4} \dot{P}_{T} d \tau+C_{1}(\tau)
$$

This implies that the first term of the r.h.s is independent of $\tau$ and $C_{1}$ is just constant because the 1.h.s. is only a function of $y$. Plugging the definition of $F$ into Eqs.(14) and (15), one has two apparently different equations

$$
\begin{aligned}
& \left(\frac{\dot{a}}{n a}\right)^{2}=-\frac{k}{a^{2}}+\left(\frac{a^{\prime}}{b a}\right)^{2}+\frac{1}{6} \kappa^{2}\left(\Lambda+\rho_{B}\right)+\frac{C_{1}(\tau)}{a^{4}} \\
& \left(\frac{\dot{a}}{n a}\right)^{2}=-\frac{k}{a^{2}}+\left(\frac{a^{\prime}}{b a}\right)^{2}+\frac{1}{6} \kappa^{2}\left(\Lambda-P_{T}\right)+\frac{1}{4} \int a^{4} \dot{P}_{T} d \tau+\frac{C_{2}(y)}{a^{4}} .
\end{aligned}
$$

\footnotetext{
${ }^{3}$ Compared with [4, 5 , $C_{1}$ is related to a free parameter $\mathcal{C}$ which may carry with the mass $M$ of $\mathrm{AdSS}_{5}$ black hole, whereas $C_{2}$ is not considered in the BDL case. In this work we will determine these by both the Einstein equation and the bulk conservation law.
} 
First we consider Eq.(18) because Eq.(17) will give us the same equation as in Eq.(18). redundant. We are in a position to account the localized distribution on the brane at $y=0$ using the Israel junction condition. From $(0,0)$-and $(i, j)$-components of the Einstein equation, we obtain

$$
\begin{aligned}
& \frac{\left[a^{\prime}\right]}{a_{0} b_{0}}=-\frac{1}{3} \kappa^{2}\left(\sigma+\rho_{b}\right), \\
& \frac{\left[n^{\prime}\right]}{n_{0} b_{0}}=\frac{1}{3} \kappa^{2}\left(-\sigma+2 \rho_{b}+3 p_{b}\right)
\end{aligned}
$$

with $\left[a^{\prime}\right] \equiv a^{\prime}\left(0^{+}\right)-a^{\prime}\left(0^{-}\right)$. On the brane we have the corresponding conservation law

$$
\dot{\rho_{b}}+3\left(\rho_{b}+p_{b}\right) \frac{\dot{a_{0}}}{a_{0}}=0
$$

where the subscript " 0 " means the location of the brane at $y=0$. Eq.(18) should also be satisfied as one approaches the brane, i.e., $y \rightarrow 0^{+}$.

$$
\left(\frac{\dot{a_{0}}}{n_{0} a_{0}}\right)^{2}=-\frac{k}{a_{0}^{2}}+\left(\frac{a^{\prime}\left(0^{+}\right)}{b_{0} a_{0}}\right)^{2}+\frac{1}{6} \kappa^{2}\left(\Lambda-P_{T}\right)+\frac{1}{4} \int a_{0}^{4} \dot{P}_{T} d t+\frac{C_{2}(0)}{a_{0}^{4}}
$$

Assuming the $Z_{2}$-symmetry of $a(-y)=a(y), n(-y)=n(y)$, we have

$$
\begin{aligned}
& \frac{a^{\prime}\left(0^{+}\right)}{b_{0} a_{0}}=-\frac{1}{6} \kappa^{2}\left(\sigma+\rho_{b}\right), \\
& \frac{n^{\prime}\left(0^{+}\right)}{b_{0} n_{0}}=\frac{1}{6} \kappa^{2}\left(-\sigma+2 \rho_{b}+3 p_{b}\right) .
\end{aligned}
$$

Redefining the time as $n_{0}(\tau, y=0) d \tau \equiv d \tau$ (i.e., $n_{0} \equiv 1$ ), one has the Friedmann-like equation on the brane

$$
H^{2} \equiv\left(\frac{\dot{a_{0}}}{a_{0}}\right)^{2}=-\frac{k}{a_{0}^{2}}+\frac{\kappa^{4} \sigma^{2}}{36}+\frac{\kappa^{2}}{6}\left(\Lambda-P_{T}\right)+\frac{1}{4} \int a_{0}^{4} \dot{P}_{T} d \tau+\frac{\kappa^{4} \sigma}{18} \rho_{b}+\frac{\kappa^{4}}{36} \rho_{b}{ }^{2}+\frac{C_{2}(0)}{a_{0}{ }^{4}} .
$$

For the fine-tuning, if one chooses $\Lambda=-\frac{1}{6} \kappa^{2} \sigma^{2}=-\frac{6}{\ell^{2} \kappa^{2}}$ with $\sigma=\frac{6}{\ell \kappa^{2}}$ then the above equation leads to

$$
H^{2}=-\frac{k}{a_{0}^{2}}-\frac{\kappa^{2}}{6} P_{T}+\frac{1}{4} \int a_{0}^{4} \dot{P}_{T} d \tau+\frac{\kappa^{2}}{3 \ell} \rho_{b}+\frac{\kappa^{4}}{36} \rho_{b}^{2}+\frac{C_{2}(0)}{a_{0}{ }^{4}} .
$$

Without the localized matter $\left(\rho_{b}=0\right)$, Eq.(26) reduces to

$$
H^{2}=-\frac{k}{a_{0}^{2}}-\frac{\kappa^{2}}{6} P_{T}+\frac{1}{4} \int a_{0}^{4} \dot{P}_{T} d \tau+\frac{C_{2}(0)}{a_{0}{ }^{4}} .
$$

Now we wish to use Eqs.(12) and (13) to obtain further information. Considering $\left(\dot{F}^{\prime}\right)=(\dot{F})^{\prime}$ and $G_{05}=0$ gives

$$
\dot{\rho}_{B} a^{\prime}+\dot{a}\left(3 \frac{a^{\prime}}{a}+\frac{n^{\prime}}{n}\right) \rho_{B}+a^{\prime} \frac{\dot{b}}{b}\left(\rho+P_{T}\right)=0 .
$$


Also from $(0,0)$-and $(i, j)$-components of the Einstein equation, one finds

$$
\rho_{B} \frac{n^{\prime}}{n}=3 P \frac{a^{\prime}}{a} .
$$

Using the above relation, Eq.(28) leads to

$$
\dot{\rho}_{B}+3\left(\rho_{B}+P+P_{T}+\frac{P}{\rho_{B}} P_{T}\right) \frac{\dot{a}}{a}+\left(\rho_{B}+P_{T}\right) \frac{\dot{b}}{b}=0 .
$$

This may be considered a variant of the bulk conservation law $\nabla_{A} \check{T}^{A}{ }_{0}=0$,

$$
\dot{\rho}_{B}+3\left(\rho_{B}+P\right) \frac{\dot{a}}{a}+\left(\rho_{B}+P_{T}\right) \frac{\dot{b}}{b}=0
$$

which can be also derived from the Bianchi identity of $\nabla_{A} G^{A}{ }_{0}=0$. If one compares Eq.(30) with Eq.(31), Eq.(30) means the violation of the conservation law due to the introduction of non-zero $P_{T}$. Actually as it stands, Eq.(30) can provide us another checkpoint to derive a consistent brane cosmology. Here we have to choose $P_{T}$ in a way that Eq.(30) is consistent with the conservation law Eq.(31).

Let us discuss some interesting cases: i) In the case of $P_{T}=P$, we find an obvious violation of conservation law. ii) If $P_{T}=0$, Eq.(30) leads to the known conservation law for the bulk matter in the brane world scenariof 四. In this case we can get the brane expansion as well as the bulk expansion. iii) $\rho_{B}=-P$ leads to the case that the brane is vacuum whereas the expansion of extra dimension is governed by $\rho_{B} \sim b^{-(1+\tilde{w})}$ according to $\dot{\rho}_{B}+\left(\rho_{B}+P_{T}\right) \frac{\dot{b}}{b}=0$ with $P_{T}=\tilde{w} \rho_{B}$. iv) If $P_{T}=-\rho_{B}$, we have the vacuum state for bulk with $\dot{\rho}_{B}=0$.

For our purpose, it is desirable to choose $P_{T}=0$. This means that the bulk matter does not flow into the fifth direction. This situation is similar to the case that $\tilde{T}^{0}{ }_{5}=0$ implies that there is no flow of a localized matter along the fifth direction. But we remind the reader that our situation is still under $\Lambda \neq 0$ and $\rho_{B} \neq 0$ in the bulk direction. Hence $P_{T}=0$ implies the matter-dominated universe in the bulk direction. In this case, comparing Eq.(18) with Eq.(17) leads to $C_{2}(0)=\kappa^{2} a_{0}^{4} \rho_{B} / 6+C_{1}$, which means that the r.h.s. is independent of $\tau$. Let us choose $C_{1}, C_{2}$ to obtain an appropriate brane cosmology (brane expansion or contraction). This means that we use two parameters $C_{1}, C_{2}$ to embed the bulk matter into the brane cosmology. Here $C_{1}=0$ for our purpose. From the fact that $C_{2}(0)=\kappa^{2} a_{0}^{4} \rho_{B} / 6$ is constant w.r.t. $\tau$, we get immediately an important relation of $\rho_{B} \sim a_{0}^{-4}$ on the brane.

On the other hand, using the bulk conservation law Eq.(31) with $P_{T}=0$, we can arrive at the same result. That is, $\rho_{B} \sim a_{0}^{-4}$ is recovered if $\dot{b}=0$ and $P=\rho_{B} / 3$. This means that the result of MDW approach can be recovered from the BDL method if the brane is an only expanding subspace in the bulk spacetime. As a result Eq.(27) leads to

$$
H^{2}=-\frac{k}{a_{0}^{2}}+\frac{\kappa^{2}}{6} \rho_{B}
$$

\footnotetext{
${ }^{4}$ Here we split the bulk tensor into the cosmological constant $\Lambda$ and the bulk fluid matter $\left(\rho_{B}, P, P_{T}\right)$. But in ref. 近, they did not introduce such a bulk matter.
} 
on the brane. Also it is important to check that from the equation (17) with $C_{1}=0$, we recover the same equation as Eq.(32).

Finally let us introduce the CFT-radiation dominated universe for one-sided brane relation? with $k=1$ [14]

$$
H^{2}=-\frac{1}{a_{0}^{2}}+\frac{8 \pi G_{4}^{N}}{3} \rho_{C F T}, \quad \rho_{C F T}=\frac{E}{V}=\frac{M \ell}{a_{0}^{4} V\left(S^{3}\right)}, \quad 8 \pi G_{4}^{N}=\frac{2 \kappa^{2}}{\ell}
$$

where $M$ is the bulk information (mass of the $\mathrm{AdSS}_{5}$ black hole on each side, while $E$ is the boundary information (its energy on the brane). $V\left(S^{3}\right)$ is the volume of the unit $S^{3}$. Comparing Eq.(32) with Eq.(33), one finds that if $\rho_{B}=2 \rho_{C F T} / \ell=2 M /\left(a_{0}^{4} V\left(S^{3}\right)\right)$, the CFT-radiation universe recovers from the BDL approach. In this case we have $C_{2}(0)=$ $\kappa^{2} \rho_{B} a_{0}^{4} / 6=\kappa^{2} M /\left(3 V\left(S^{3}\right)\right)$. This implies that $C_{2}(0) \sim M$. If $C_{2}=0$, the bulk spacetime is the exact $\mathrm{AdS}_{5}$ spacetime with $\Lambda . C_{2} \neq 0$ is related to the initial bulk matter distribution : the 5D non-vanishing Weyl tenor. In the exact $\mathrm{AdS}_{5}$ space, we find the vanishing Weyl tensor, whereas we get the non-vanishing one for the $\mathrm{AdSS}_{5}$ black hole spacetime.

Let us discuss what conclusions can follow from Eq.(33). This implies that Friedmann equation knows about thermodynamics of the radiation-matter CFT on the brane. Initially the Friedmann equation has nothing to do with the CFT. But using both the AdS/CFT correspondence and the various entropy bounds, we make a connection between the above Friedmann equation and the $\operatorname{entropy}(S)$-energy $(E)$ relation for the CFT [12,14. Explicitly we arrive from Eq.(33) exactly at the Cardy-Verlinde formula : $S=\left(2 \pi a_{0} / 3\right) \sqrt{E_{c}\left(2 E-E_{c}\right)}$ with the Casimir energy $E_{c}$, when the brane crosses the horizon of the balck hole.

In conclusion we establish the holographic principle using the BDL approach. We get the Friedmann equation which describes the CFT radiation-dominated universe on the brane from the initial bulk consideration. Especially we use two parameters $C_{1}$ and $C_{2}$ in the BDL approach to embed the initial bulk configuration of the $\mathrm{AdSS}_{5}$ black hole into the brane. With $\rho_{B} \sim M / a_{0}^{4}$, the bulk matter satisfies $P=\rho_{B} / 3$ on the brane. This means that the black hole with mass $M$ in the BDL method provides the CFT radiation matter on the brane as in the case of the moving domain wall approach. The BDL (MDW) approaches use the Gaussian normal (Schwarzschild) coordinates. Mukhoyama et al. showed that although there exist apparent different configurations, all of two-types solutions represent the same spacetime described with different coordinate systems [10]. Here our calculation also shows that two approaches give us the same result. In this sense our BDL approach can be considered as the domain wall approach fixed at $y=0$, in compared with the MDW approach.

\section{ACKNOWLEDGMENTS}

We thank R.G. Cai for helpful discussions. This work was supported in part by the Brain Korea 21 Program, Ministry of Education, Project No. D-0025 and KOSEF, Project No.

\footnotetext{
${ }^{5}$ The relevant difference between one-sided and two-sided scenarios is to define the $4 \mathrm{D}$ Newtonian constant [7]: $8 \pi G_{4}^{N}=\frac{2 \kappa^{2}}{\ell}$ for one-sided , $8 \pi G_{4}^{N}=\frac{\kappa^{2}}{\ell}$ for two-sided.
} 
2000-1-11200-001-3. 


\section{REFERENCES}

[1] L. Randall and R. Sundrum, Phys. Rev.Lett. 83 (1999) 3370 [hep-ph/9905221.

[2] L. Randall and R. Sundrum, Phys. Rev.Lett. 83 (1999) 4690 [hep-th/9906064].

[3] P. Binetruy, C. Deffayet and D. Langlois, Nucl. Phys. B 565 (2000) 269 [hep-th/9905012.

[4] P. Binetruy, C. Deffayet, U. Ellwanger and D. Langlois, Phys. Lett. B 477 (2000) 285 hep-th/9910219.

[5] C. Deffayet, hep-th/0010186; T. Shiromizu and D. Ida, hep-th/0102035.

[6] H. B. Kim and H. D. Kim, Phys. Rev. D61 (2000 )064003 hep-ph/9909053.

[7] M. J. Duff and T. Liu, Phys. Rev. Lett. 85 (2000) 2052.

[8] P. Kraus, JHEP 9912 (1999) 011 hep-th/9910149]; D. Ida, JHEP 0009 (2000) 014; H. Collins and B. Holdom, hep-ph/0003173; H. Stoica, S. H. H. Tye and I. Wasserman, hep-th/0004126; C. Barcelo and M. Visser, hep-th/0004056; A. C. Davis, I. Vernon, S. C. Davis and W. B. Perkins, hep-ph/0008132; N. J. Kim, H. W. Lee, and Y. S. Myung, hep-th/0101091.

[9] W. Israel, Nuovo Cim. B44 (1966) 1; ibid. B48 (1967) 463.

[10] S. Mukhoyama, T. Shiromizu and K. Maeda, hep-th/9912287.

[11] N. Deruelle and T. Dolezel, gr-qc/0004021.

[12] E. Verlinde, hep-th/0008140.

[13] R. Brustein, S. Foffa and G. Veneziano, hep-th/0101083; D. Klemm, A. Petkou and G. Siopsis, hep-th/0101076; B. Wang, E. Abdalla and R. K. Su, hep-th/0101073; S. Nojiri and S. Odintsov, hep-th/0011115; F.L. Lin, hep-th/0010127; D. Kutasov and F. Larsen, hep-th/0009244; R. G. Cai, hep-th/0102113; Y.S. Myung, hep-th/0102184; D. Birmingham and S. Mokhtari, hep-th/0103108; S. Nojiri and S. Odintsov, hep-th/0103078; L. Anchordoqui, C. Nunez, K. Olsen, hep-th/0007064 ; A. K. Biswas and S. Mukherji, hep-th/0102138; Y.S. Myung, hep-th/0103241.

[14] I. Savonije and E. Verlinde, hep-th/0102042. 